\title{
STABLE APPROXIMATIONS OF A MINIMAL SURFACE PROBLEM WITH VARIATIONAL INEQUALITIES
}

\author{
M. ZUHAIR NASHED AND OTMAR SCHERZER
}

\begin{abstract}
In this paper we develop a new approach for the stable approximation of a minimal surface problem associated with a relaxed Dirichlet problem in the space $\mathrm{BV}(\Omega)$ of functions of bounded variation. The problem can be reformulated as an unconstrained minimization problem of a functional $\mathcal{J}$ on $\operatorname{BV}(\Omega)$ defined by $\mathcal{J}(u)=\mathcal{A}(u)+\int_{\partial \Omega}|T u-\phi|$, where $\mathcal{A}(u)$ is the "area integral" of $u$ with respect to $\Omega, T$ is the "trace operator" from $\mathrm{BV}(\Omega)$ into $L^{1}(\partial \Omega)$, and $\phi$ is the prescribed data on the boundary of $\Omega$. We establish convergence and stability of approximate regularized solutions which are solutions of a family of variational inequalities. We also prove convergence of an iterative method based on Uzawa's algorithm for implementation of our regularization procedure.
\end{abstract}

\section{INTRODUCTION}

The minimal surface problem or the problem of Plateau is one of the oldest problems in the Calculus of Variations. See for example [19] (pp. 72-73). In the classical and simplest version, given a regular bounded domain $\Omega \subseteq \mathbb{R}^{2}$ with smooth boundary $\partial \Omega$ and prescribed smooth boundary function (data) $\phi$, we seek a function $u \in C^{1}(\bar{\Omega})$ which has these boundary values and

1991 Mathematics Subject Classification. Primary 49J40, 49Q05, 49N60, 49J45, 65K10; secondary 26A45, 65J15, 65J20.

Key words and phrases. Minimal surface problem, relaxed Dirichlet problem, nondifferentiable optimization in nonreflexive spaces, variational inequalities, bounded variation norm, Uzawa's algorithm.

The work of the second author was supported by the Austrian Fonds zur Förderung der wissenschaftlichen Forschung, Grant J01088-TEC, while he was on leave from Institut für Mathematik, Universität Linz, Altenberger Str. 69, A-4040 Linz, Austria.

Received: February 19, 1997. 
minimizes the surface area functional

$$
S(u)=\int_{\Omega} \sqrt{1+|\nabla u|^{2}} .
$$

Letting $\Gamma:=\left\{u \in C^{1}(\bar{\Omega}): u=\phi\right.$ on $\left.\partial \Omega\right\}$ and $\Gamma_{0}:=\left\{h \in C^{1}(\bar{\Omega}): h=\right.$ 0 on $\partial \Omega\}$, it is easy to show that the functional $S$ is strictly convex on $\Gamma$; thus seeking the unique minimizer of $S$ on $\Gamma$ is equivalent to solving the variational equality $\delta S(u ; h)=0$ for all $h \in \Gamma_{0}$, where $\delta S(u ; h)$ is the Gateaux variation of $S$ at $u$ in the direction $h$. If the set of admissible minimizers is taken to be $\Gamma \cap C^{2}(\Omega)$, then a standard argument using Green's theorem shows that the minimizer of (1) satisfies the minimal surface equation

$$
\left(1+u_{y}^{2}\right) u_{x x}-2 u_{x} u_{y} u_{x y}+\left(1+u_{x}^{2}\right) u_{y y}=0 .
$$

Let $W$ be a subset of a vector space $V$. Recall that a functional $S$ on $W$ has a one-sided Gateaux variation at $u_{0} \in W$ if for each $h \in V$ for which $u_{0}+t h \in W$ for sufficiently small positive $t$

$$
\delta^{+} S\left(u_{0} ; h\right)=\lim _{t \rightarrow 0^{+}} \frac{S\left(u_{0}+t h\right)-S\left(u_{0}\right)}{t}
$$

exists in the extended real numbers. Suppose the functional $S$ has a onesided Gateaux variation at a point $u_{0} \in W$. Then a necessary condition for $S$ to have a minimum at $u_{0}$ is that

$$
\delta^{+} S\left(u_{0} ; u-u_{0}\right) \geq 0
$$

for all $u \in V$ for which $\delta^{+} S\left(u_{0} ; u-u_{0}\right)$ exists. For convex functionals, condition (2) is also sufficient. Thus a necessary and sufficient condition for $u_{0}$ to minimize a convex functional $S$ is that $u_{0}$ satisfies the variational inequality (2). Without additional "regularity" assumptions on the domain $W$ and the functional $S$, it is not possible to derive an equation or even a variational equality that a minimizer satisfies. If $W$ is a subspace (a kind of regularity on the domain), then clearly the variational inequality (2) becomes a variational equality. Furthermore, under some smoothness assumptions (regularity) on the functional $S$ it is usually possible to show that the variational equality is equivalent to an equation involving $u_{0}$ only. All this is clearly illustrated by the above discussion of the classical minimal surface problem.

In recent years there has been considerable interest in a variety of minimal surface problems for which the classical theory of the calculus of variations does not apply and the types of regularity mentioned above are not satisfied. This is the situation in the setting of this paper. 
Recent developments in minimal surface problems are along three directions. In one direction the aims have been to treat the problem of Plateau in its full generality using measure-theoretic methods, to present a theory of minimal hypersurfaces in arbitrary dimension and for arbitrary boundary data, and to treat global/topological aspects. For some results and perspectives on these developments see the books of Giusti [8], Nitsche [17], Osserman [18], Fomenko [7], and the papers by Ball [1], Ball and Murat [2], Jenkins and Serrin [12], Nitsche [16] and related references cited therein. Of particular relevance to this paper are the advances culminating in the treatment of the Dirichlet problem for the minimal surface equation in its relaxed formulation. For an excellent treatment of this formulation see Giusti [8]. This required the reformulation of that problem as an unconstrained minimization problem on the space of functions of bounded variation, thereby posing an interesting optimization problem in nonreflexive Banach spaces, for which standard optimization theory (developed principally for reflexive Banach spaces) does not apply. The second direction of recent developments in minimal surface problems focuses on numerical (approximate) treatment (including numerical experiments and computer-aided discovery of minimal surfaces) for both the classical version and along the extensions described above. For some results and perspectives on these topics see Jouron [13], Concus [3], Hoffman [11], Dacorogna [4], Ekeland and Temam [5], and related references cited therein. The third direction is concerned with applications, particularly to soap films, crystals and optimal design (see for example, Hildebrandt and Tromba [10]), and to elasticity (see for example, Ball [1]).

The purpose of this paper is to show that the framework and theory of stable approximations of nondifferentiable optimization problems in nonreflexive Banach spaces, developed recently by the authors [15], can be adapted to provide an algorithm for stable approximations for the relaxed Dirichlet problem for a minimal surface problem.

To put some key issues in perspective we make some remarks that highlight differences between optimization theory in reflexive and nonreflexive Banach spaces.

Optimization theory (existence, uniqueness, and stability) in nonreflexive Banach spaces is more subtle than the theory in reflexive Banach spaces. This is partly due to the fact that the weak topology of a Banach space does not coincide with the weak-star topology of its dual. As a consequence the Alaoglu-Bourbaki-Kakutani theorem and the Mazur theorem in functional analysis, which are central to the role that the weak topology plays for 
optimization theory in reflexive Banach spaces, have no direct relevance for optimization in nonreflexive spaces. Another marked difference arises in consideration of stability of minimizing sequences. We indicate this by the following situation, which is important to the framework of this paper: For a function $f \in \mathrm{BV}(\Omega)$ (the space of functions of bounded variation) it cannot be expected (in general) to find functions $f_{j} \in C^{\infty}(\Omega)$ such that $f_{j} \rightarrow f$ in $L^{1}(\Omega)$ and $\int_{\Omega}\left|D\left(f_{j}-f\right)\right| \rightarrow 0$, where $D$ denotes "differentiation". This is due to the fact that the closure of the $C^{\infty}$-functions with respect to the BV-norm is the Sobolev space $W^{1,1}(\Omega)[8]$. This shows that functions of bounded variation, which are not in $W^{1,1}(\Omega)$ (like piecewise constant functions), cannot be approximated by a family of smooth functions with respect to the bounded variation norm.

Existence and uniqueness of solutions of various classes of optimization problems in nonreflexive Banach spaces have been studied in the literature. However, questions of stability with respect to the input data have not been satisfactorily addressed, partly due to the difficulty in the situation described above. To overcome this difficulty, stability questions can be considered in "weaker" norms. This approach is often unsatisfactory when the solution to be approximated is smooth, as it is the case in the minimal surface problem (see Proposition 3.1). A more satisfactory approach from the point of stable approximations is to consider a family of modified functionals on Hilbert spaces (which are appropriately embedded in the nonreflexive Banach space) and to consider the minimizers of the modified functionals as approximate solutions of the original problem. This idea was recently used by the authors to study optimization methods in nonreflexive Banach spaces [15].

In Section 2 we summarize results which are minor modifications of those in [15], specifically adapted for application to a minimal surface problem. In Section 3 we develop a new approach for the stable approximation of a minimal surface problem associated with a relaxed Dirichlet problem as formulated in Giusti [8], and establish convergence and stability of this procedure based on the results in Section 2. In Section 4 we prove convergence of an iterative implementation for the stable numerical procedure developed in Section 3.

\section{Nonsmooth OPtimizATION IN NONREFLEXIVE SPACES}

Motivated by a class of inverse problems (source identification and image restoration) the authors have recently studied stable methods for approximating solutions of optimization problems in nonreflexive Banach spaces. In 
this section we consider a variant of the setting and results in [15], adapted for applicability to a minimal surface problem considered in this paper. We omit the proofs since they follow the same arguments as in [15]. Let $\mathcal{D}$ and $Y$ be (not necessarily reflexive) Banach spaces, $\Phi: \mathcal{D} \times Y \rightarrow \mathbb{R}$, and $k: \mathcal{D} \rightarrow \mathbb{R}$ be nonlinear (not necessarily differentiable) functionals satisfying certain conditions (see below). The space $Y$ will be referred to as the data space. For a given perturbed data $\phi^{\delta}$ of the exact data $\phi$, we consider the following minimization problem:

$$
\min _{v \in \mathcal{D}} \mathcal{J}(v), \text { where } \mathcal{J}(v):=\Phi\left(v, \phi^{\delta}\right)+k(v) .
$$

We associate with the problem (3) a family of "regularized" minimization problems on real Hilbert spaces $\mathcal{D}_{\varepsilon}$ (with norms $\|\cdot\|_{\mathcal{D}_{\varepsilon}}$ and inner products $\left.(., .)_{\mathcal{D}_{\varepsilon}}\right)$ :

$$
\min _{v \in \mathcal{D}_{\varepsilon}} \mathcal{J}_{\varepsilon}(v), \text { where } \mathcal{J}_{\varepsilon}(v)=\Phi\left(v, \phi^{\delta}\right)+k_{\varepsilon}(v)+\frac{1}{2} e(\varepsilon)\|v\|_{\mathcal{D}_{\varepsilon}}^{2},
$$

where $e(\varepsilon)>0$, such that for each vector $\varepsilon$ of positive numbers the problem (4) has a unique solution which, moreover, can be characterized by a variational inequality. The goal of this section is two-fold:

- to develop tractable sufficient conditions under which these two requirements are met, and

- to establish convergence of the approximate solutions to a solution of the original minimization problem.

Note that the family of regularized optimization problems (4) entails modifications of both the functional $\mathcal{J}$ and the domain $\mathcal{D}$. The exact nature of these modifications is given below, and is tailored to achieve the two objectives stated above.

Existence and Uniqueness. It is well known that for fixed vector $\varepsilon$ of positive numbers, the problem (4) has a unique minimizer (denoted by $u$ ), which can be characterized as the solution of the variational inequality

$$
\begin{gathered}
e(\varepsilon)(v, v-u)_{\mathcal{D}_{\varepsilon}}+\Phi\left(v, \phi^{\delta}\right)+k_{\varepsilon}(v)-\Phi\left(u, \phi^{\delta}\right)-k_{\varepsilon}(u) \geq 0 \\
\text { for all } v \in \mathcal{D}_{\varepsilon},
\end{gathered}
$$

if $\mathcal{J}_{\varepsilon}(v)$ is a weakly lower semicontinuous, proper convex functional on $\mathcal{D}_{\varepsilon}$. For a proof see, for example, Section 4 of Chapter 1 in [9]. 
Stability. The next theorem provides sufficient conditions under which the solutions of the optimization problem (4) are stable with respect to perturbations in the data $y$.

Theorem 2.1. Let $\varepsilon$ be a vector of fixed positive numbers, and write $\varepsilon>0$. Assume that

1. $\mathcal{J}_{\varepsilon}(v)$ is a weakly lower semicontinuous, proper convex functional on $\mathcal{D}_{\varepsilon}$.

2. $k_{\varepsilon}$ and $\Phi$ are positive functionals.

3. The functional $\Phi$ is Lipschitz continuous with respect to the data $\phi$, i.e., for all $w \in \mathcal{D}_{\varepsilon}$

$$
\left|\Phi(w, \phi)-\Phi\left(w, \phi^{\delta}\right)\right| \leq C_{1, \varepsilon} \delta, \quad \text { for }\left\|\phi-\phi^{\delta}\right\| \leq \delta .
$$

Then each minimizing sequence $\left\{u_{\varepsilon}^{\delta_{n}}\right\}$ of the functional in (4) (where in (4) $\phi^{\delta}$ is replaced by $\phi^{\delta_{n}}$ and $\left.\left\|\phi^{\delta_{n}}-\phi\right\| \leq \delta_{n}\right)$ converges as $\delta_{n} \rightarrow 0$ to a minimizing element of the problem (4) (where now in (4) $\phi^{\delta}$ is replaced by $\phi$ ).

(Weak) Convergence. We next state additional conditions under which the minimizers of the functionals in (4), which have been shown to be stable with respect to perturbations in the data $\phi$, also provide approximate solutions of the original minimization problem (3), and so the family of minimization problems (4) provides a regularization method:

4. The Hilbert spaces $\mathcal{D}_{\varepsilon}$ have continuous embeddings in the (nonreflexive) Banach space $\mathcal{D}$, and $\mathcal{D}$ itself has a compact embedding in some reflexive Banach space $\mathcal{Z}$.

5. For all $w \in \mathcal{D}, \phi, \phi^{\delta} \in Y$ satisfying $\left\|\phi^{\delta}-\phi\right\| \leq \delta$

$$
\left|\Phi(w, \phi)-\Phi\left(w, \phi^{\delta}\right)\right| \leq C_{1} \delta .
$$

6. The minimum of the functional $\mathcal{J}$ is attained at $\underline{u} \in \mathcal{D}$.

7. There exist sequences $\varepsilon_{n}>0, \eta_{n} \in \mathcal{D}_{\varepsilon_{n}}$, with the properties

$$
\begin{aligned}
e\left(\varepsilon_{n}\right)\left\|\eta_{n}\right\|_{\mathcal{D}_{\varepsilon_{n}}}^{2} & \rightarrow 0, \\
\limsup _{n \in \mathbb{N}}\left\{k_{\varepsilon_{n}}\left(\eta_{n}\right)+\Phi\left(\eta_{n}, \phi\right)\right\} & \leq k(\underline{u})+\Phi(\underline{u}, \phi) .
\end{aligned}
$$

8. For all vectors $\varepsilon$ of positive numbers and all $u \in \mathcal{D}_{\varepsilon}$

$$
\|u\|_{\mathcal{D}} \leq C_{2} \mathcal{J}_{\varepsilon}(u)+D_{2} .
$$

9. For given data $\phi$ the functional

$$
\Phi(v, \phi)+k(v)
$$


is lower semicontinuous on $\mathcal{D}$ with respect to the norm on $\mathcal{Z}$, i.e., if $v_{n} \in \mathcal{D}$ and $v_{n} \rightarrow v$ in $\mathcal{Z}$, then $v \in \mathcal{D}$, and

$$
\Phi(v, \phi)+k(v) \leq \liminf \left\{\Phi\left(v_{n}, \phi\right)+k\left(v_{n}\right)\right\} .
$$

Theorem 2.2. Let assumptions 1 - 9 be satisfied. Then each minimizing sequence $\left\{u_{\varepsilon_{n}}^{\delta_{n}}\right\}$ of $\mathcal{J}_{\varepsilon}$ has a subsequence which converges as $\delta_{n} \rightarrow 0$ to a minimizing element of $\mathcal{J}$ with respect to the norm on $\mathcal{Z}$. If the minimizer of $\mathcal{J}$ is unique, then $\left\{u_{\varepsilon_{n}}^{\delta_{n}}\right\}$ is convergent.

3. A minimal surface Problem and a Stable PRocedure For its APPROXIMATE SOLUTION

In this section, we apply the general result of Section 2 to an algorithm for the stable approximation of the following minimal surface problem:

Let $\Omega \subseteq \mathbb{R}^{d}, d \geq 2$ with $C^{1}$-boundary $\partial \Omega$. We define the space $\operatorname{BV}(\Omega)$ of functions of bounded variation on $\Omega$ by

$$
\begin{aligned}
& \operatorname{BV}(\Omega)=\left\{u \in L^{1}(\Omega):\right. \\
& \int_{\Omega}|D u|:=\sup \left\{\int_{\Omega} u \nabla \cdot g: g=\left(g_{1}, \ldots, g_{d}\right) \in C_{0}^{1}\left(\Omega, \mathbb{R}^{d}\right),\right. \\
& \left.\left.|g(x)|^{2}=\sum_{i=1}^{d} g_{i}^{2}(x) \leq 1, x \in \Omega\right\}<\infty\right\} .
\end{aligned}
$$

It is easy to see that we can use in the definition of $\mathrm{BV}(\Omega)$ the following equivalent definition of $\int_{\Omega}|D u|$

$$
\begin{gathered}
\int_{\Omega}|D u|:=\sup \left\{-\int_{\Omega} u \nabla \cdot g: g=\left(g_{1}, \ldots, g_{d}\right) \in C_{0}^{1}\left(\Omega, \mathbb{R}^{d}\right),\right. \\
\left.|g(x)|^{2}=\sum_{i=1}^{d} g_{i}^{2}(x) \leq 1, x \in \Omega\right\} .
\end{gathered}
$$

Following Giusti [8], we define for $u \in \mathrm{BV}(\Omega)$ the area integrand of $u$ with respect to $\Omega$ by

$$
\mathcal{A}(u):=\int_{\Omega} \sqrt{1+|D u|^{2}} d x,
$$


where

$$
\begin{aligned}
& \int_{\Omega} \sqrt{1+|D u|^{2}} d x \\
& :=\sup \left\{\int_{\Omega}\left(g_{d+1}+u \sum_{i=1}^{d} g_{i}\right) d x:\right. \\
& \left.\quad g=\left(g_{1}, \ldots, g_{d+1}\right) \in C_{0}^{1}\left(\Omega, \mathbb{R}^{d+1}\right),|g| \leq 1\right\} .
\end{aligned}
$$

The Dirichlet problem for the minimal surface problem is to find a function $u$ of minimal area $\mathcal{A}(u)$, as defined in $(6)-(7)$, in the class $\operatorname{BV}(\Omega)$ with prescribed data $\phi$ on $\partial \Omega$.

For the minimal surface problem associated with $(6)-(7)$, it is shown in Section 4 of Chapter 14 in [8] that the solution of the Dirichlet problem for this minimal surface problem can be reformulated in terms of an unconstrained minimization problem of the functional $\mathcal{J}$ defined on $\mathcal{D}:=\mathrm{BV}(\Omega)$ by

$$
\mathcal{J}(u):=\mathcal{A}(u)+\int_{\partial \Omega}|T u-\phi|
$$

where $T$ is the trace operator from $\mathrm{BV}(\Omega)$ into $L^{1}(\partial \Omega)$. For a precise definition of the trace operator in this setting see Evans and Gariepy [6]. This definition requires major technical modifications of the "classical" notion of trace, since mean values of a function have to replace point evaluations.

Proposition 3.1. (Proposition 14.3 in [8]) Let $\phi \in L^{1}(\partial \Omega)$. Then

$$
\inf \{\mathcal{A}(u): u \in B V(\Omega), u=\phi \text { on } \partial \Omega\}=\inf \{\mathcal{J}(u): u \in B V(\Omega)\} \text {. }
$$

In Theorem 14.5 in [8] it is shown that $\mathcal{J}(u)$ attains its infimum on BV $(\Omega)$. Together with Proposition 3.1 this establishes the existence of a solution of the Dirichlet problem for the minimal surface problem. In [8] (see pp. 169-171) it is also shown that the solution of the Dirichlet problem for the minimal surface problem is locally smooth, which especially guarantees that the minimizing element of $\mathcal{J}(u)$ is in $W^{1,1}(\Omega)$. The following proposition follows immediately from these observations:

Proposition 3.2. Let $\phi \in L^{1}(\partial \Omega)$. Then

$$
\inf \left\{\mathcal{J}(u): u \in W^{1,1}(\Omega)\right\}=\inf \{\mathcal{J}(u): u \in B V(\Omega)\}
$$


Based on these considerations the numerical solution of the Dirichlet problem for the minimal surface problem with a finite element method was proposed by Jouron [13], where it is shown that the finite element approximations converge with respect to the $W^{1,1}(\Omega)$-norm. In this paper we are concerned with continuous dependence of the solution of the minimal surface problem on the input data $\phi^{\delta}$; e.g. these considerations are important if measurement errors in the data $\phi$ have to be taken into account. Based on the theoretical results in Section 2 an approximation $\mathcal{J}_{\varepsilon}$ of $\mathcal{J}$ is introduced, which allows stable calculation of the minimizing elements, and for appropriately chosen parameter $\varepsilon$ we prove that the approximate solutions converge to a solution of the minimal surface problem.

Let $\varepsilon=\left(\varepsilon_{1}, \varepsilon_{2}\right)$, where $\varepsilon_{1}, \varepsilon_{2}$ are positive numbers. Consider the functional $\mathcal{J}_{\varepsilon}: W^{2, s}(\Omega) \rightarrow \mathbb{R}$ defined by

(8) $\mathcal{J}_{\varepsilon}(v)=\frac{\varepsilon_{1} \varepsilon_{2}}{2}\|v\|_{W^{2, s}(\Omega)}^{2}+\varepsilon_{1}\|v\|_{L^{1}(\Omega)}+\int_{\Omega} \sqrt{1+|\nabla v|^{2}}+\int_{\partial \Omega}\left|T v-\phi^{\delta}\right|$;

here, as usual, $W^{2, s}(\Omega)(s \in \mathbb{N})$ denotes the Sobolev space of functions with square integrable derivatives up to order $s$.

Notation 1 In order to use the general results in Section 2 we first reformulate the minimal surface problem and its approximations in the framework of Section 2. To this end, let

- $\mathcal{D}:=\operatorname{BV}(\Omega), \mathcal{D}_{\varepsilon}:=W^{2, s}(\Omega), \mathcal{Z}:=L^{p}(\Omega), Y:=L^{1}(\partial \Omega)$, where $1 \leq p<$ $\frac{d}{d-1}$.

- $e(\varepsilon):=\varepsilon_{1} \varepsilon_{2}$.

- $k(v):=\int_{\Omega} \sqrt{1+|\nabla v|^{2}}, \quad k_{\varepsilon}(v):=\int_{\Omega} \sqrt{1+|\nabla v|^{2}}+\varepsilon_{1} \int_{\Omega}|v|$.

- $\Phi\left(v, \phi^{\delta}\right):=\int_{\partial \Omega}\left|T v-\phi^{\delta}\right|$.

Before we prove stability and convergence of the minimizers of the functional $\mathcal{J}_{\varepsilon}\left(\phi^{\delta}\right)$ we introduce some notation: Let $B_{R}$ be a sphere of radius $R$ surrounding $\Omega$, let $u \in W^{1,1}(\Omega)$, let $\bar{\phi} \in W^{1,1}\left(B_{R} \backslash \Omega\right)$ be an extension of $\phi \in L^{1}(\partial \Omega)$ (for the existence of such an extension see [8]) and define

$$
\hat{u}=u \text { in } \Omega \text { and } \hat{u}=\bar{\phi} \text { in } B_{R} \backslash \Omega \text {. }
$$

Then $\hat{u} \in \operatorname{BV}\left(B_{R}\right)$ (see [8]). Analogously it can be shown that $\hat{\phi}$ defined by

$$
\hat{\phi}=0 \text { in } \Omega, \quad \hat{\phi}=\bar{\phi} \text { in } B_{R} \backslash \Omega
$$


is an element of $\mathrm{BV}\left(B_{R}\right)$. From the definition of $\mathcal{A}$ (applied to $B_{R}$ instead of $\Omega$ ) it follows that

$$
\int_{B_{R}} \sqrt{1+|D(\hat{u}-\hat{\phi})|^{2}}=\int_{\Omega} \sqrt{1+|\nabla u|^{2}}+\int_{\partial \Omega}|T u-\phi| .
$$

We next use these observations to prove the following weak lower semicontinuity results:

Lemma 3.3. Let $u_{n} \in B V(\Omega), u \in L^{1}(\Omega)$, such that $u_{n} \rightarrow u$ in $L^{1}(\Omega)$. Then $u \in B V(\Omega)$. Let $\hat{u}_{n}$ and $\hat{u}$ be defined as above. Then

$$
\int_{B_{R}} \sqrt{1+|D(\hat{u}-\hat{\phi})|^{2}} \leq \liminf \int_{B_{R}} \sqrt{1+\left|D\left(\hat{u}_{n}-\hat{\phi}\right)\right|^{2}} .
$$

In particular, if $u_{n} \in W^{1,1}(\Omega)$, then

$$
\int_{B_{R}} \sqrt{1+|D(\hat{u}-\hat{\phi})|^{2}} \leq \liminf \left\{\int_{\Omega} \sqrt{1+\left|\nabla u_{n}\right|^{2}}+\int_{\partial \Omega}\left|T u_{n}-\phi\right|\right\} .
$$

If additionally $u \in W^{1,1}(\Omega)$, then

$$
\int_{\Omega} \sqrt{1+|\nabla u|^{2}}+\int_{\partial \Omega}|T u-\phi| \leq \liminf \left\{\int_{\Omega} \sqrt{1+\left|\nabla u_{n}\right|^{2}}+\int_{\partial \Omega}\left|T u_{n}-\phi\right|\right\} .
$$

Proof. Since

$$
\begin{aligned}
\int_{\Omega}|D u| & =\sup \left\{\int_{\Omega} u \nabla \cdot g: g=\left(g_{1}, \ldots, g_{d}\right) \in C_{0}^{1}\left(\Omega, \mathbb{R}^{d}\right),|g| \leq 1\right\} \\
& =\sup \left\{\lim _{n \rightarrow \infty} \int_{\Omega} u_{n} \nabla \cdot g: g=\left(g_{1}, \ldots, g_{d}\right) \in C_{0}^{1}\left(\Omega, \mathbb{R}^{d}\right),|g| \leq 1\right\},
\end{aligned}
$$

it follows that

$$
\int_{\Omega}|D u| \leq \liminf _{n \in \mathbb{N}} \int_{\Omega}\left|D u_{n}\right|,
$$

which proves that $u \in \mathrm{BV}(\Omega)$. The remaining assertions follow from the remarks preceding Lemma 3.3.

Using Lemma 3.3 we next prove that the minimizers of the functional $\mathcal{J}_{\varepsilon}$ defined in (8) are stable with respect to perturbations of the data $\phi$.

Theorem 3.4. Let $\varepsilon=\left(\varepsilon_{1}, \varepsilon_{2}\right)$ where $\varepsilon_{1}, \varepsilon_{2}$ are positive numbers.

- For each $\phi^{\delta} \in L^{1}(\partial \Omega)$, there exists a unique element $u_{\varepsilon}\left(\phi^{\delta}\right) \in W^{2, s}(\Omega)$ which minimizes the functional $\mathcal{J}_{\varepsilon}$, defined in (8).

- If $\phi^{\delta} \rightarrow \phi$ in $L^{1}(\partial \Omega)$, then $u_{\varepsilon}\left(\phi^{\delta}\right) \rightarrow u_{\varepsilon}(\phi)$ in $W^{2, s}(\Omega)$. 
Proof. For a fixed $\phi^{\delta}$, the existence and uniqueness of the minimizer of $\mathcal{J}_{\varepsilon}$ follow immediately from the general results in Section 2. Since $W^{2, s}(\Omega)$ can be compactly embedded into $L^{1}(\Omega)$, it follows from Lemma 3.3 that $\mathcal{J}_{\varepsilon}$ is weakly lower semicontinuous on $W^{2, s}(\Omega)$. Finally, the stability of the minimizers of $\mathcal{J}_{\varepsilon}$ with respect to data perturbations follows from Theorem 2.1.

Under the general assumptions of this section it is not known if the solution of the minimal surface problem is necessarily unique. However, any two solutions must differ by a constant (see Proposition 14.12 in [8]).

For the rest of this section we consider minimal $L^{1}$-norm (briefly minimal) solutions of the minimal surface problem.

Definition 3.5. Let $d=\inf \{\mathcal{J}(u): u \in B V(\Omega)\}$. An element $\underline{u}$ is called a minimal ( $L^{1}-$ norm) solution of the minimal surface problem if it satisfies

$$
\begin{aligned}
\mathcal{J}(\underline{u}) & =d \\
\|\underline{u}\|_{L^{1}(\Omega)} & \leq\|u\|_{L^{1}(\Omega)} \text { for all } u \text { which satisfy } \mathcal{J}(u)=d .
\end{aligned}
$$

Lemma 3.6. There exists a minimal solution of the minimal surface problem.

Proof. Let $u_{0}$ be any solution of the minimal surface problem. Then

$$
\{u: \mathcal{J}(u)=d\} \subseteq\left\{u_{0}+c: c \in \mathbb{R}\right\} .
$$

Let $c_{n}$ be a sequence of real numbers such that

$$
\left\|u_{0}+c_{n}\right\|_{L^{1}(\Omega)} \rightarrow \inf .
$$

From triangle inequality,

$\left|c_{n}\right|$ meas $(\Omega) \leq\left\|c_{n}+u_{0}\right\|_{L^{1}(\Omega)}+\left\|u_{0}\right\|_{L^{1}(\Omega)} \leq \inf +\left\|u_{0}\right\|_{L^{1}(\Omega)}<\infty$ for $n \rightarrow \infty$.

Therefore the sequence $\left\{c_{n}\right\}$ is bounded in $\mathbb{R}$ and consequently has a convergent subsequence, which will be again denoted by $\left\{c_{n}\right\}$, i.e., $c_{n} \rightarrow c$, and therefore

$d=\liminf _{n \in \mathbb{N}}\left\{\mathcal{A}\left(u_{0}+c_{n}\right)+\int_{\partial \Omega}\left|u_{0}+c_{n}-\phi^{\delta}\right|\right\}=\mathcal{A}\left(u_{0}+c\right)+\int_{\partial \Omega}\left|u_{0}+c-\phi^{\delta}\right|$,

and

$$
\left\|u_{0}+c_{n}\right\|_{L^{1}(\Omega)} \rightarrow\left\|u_{0}+c\right\|_{L^{1}(\Omega)} \text { for } n \rightarrow \infty .
$$

Consequently $u_{0}+c$ is a minimal solution of the minimal surface problem.

To prove that the family of functionals $\mathcal{J}_{\varepsilon}$ provides a regularization method in the sense of Theorem 2.2, we use the following lemma: 
Lemma 3.7. Let $\underline{u} \in W^{1,1}(\Omega)$. Let $\left\{\rho_{n}\right\}$ be a monotonically decreasing sequence of positive numbers that converges to zero. Then there exists a sequence $\left\{\eta_{n}\right\} \in W^{2, s}(\Omega)$ which satisfies:

- $\int_{\Omega}\left|\eta_{n}-\underline{u}\right| \leq \frac{\rho_{n}}{2}$.

- $\left|\int_{\Omega} \sqrt{1+\left|\nabla \eta_{n}\right|^{2}}-\int_{\Omega} \sqrt{1+|\nabla \underline{u}|^{2}}\right| \leq \frac{\rho_{n}}{2}$.

- $\int_{\partial \Omega}\left|T \eta_{n}-T \underline{u}\right| \leq \frac{\rho_{n}}{2}$.

Proof. Since $C^{\infty}(\bar{\Omega})$ is dense in $W^{1,1}(\Omega)$ and since the trace operator $T$ from $W^{1,1}(\Omega)$ into $L^{1}(\partial \Omega)$ is continuous (see e.g. [6]), there exists a sequence $\left\{\eta_{n}\right\} \in C^{\infty}(\bar{\Omega})$ such that $\left\|\eta_{n}-\underline{u}\right\|_{W^{1,1}(\Omega)} \leq \frac{\rho_{n}}{2}$ and $\left\|T \eta_{n}-T \underline{u}\right\|_{L^{1}(\partial \Omega)} \leq \frac{\rho_{n}}{2}$. The second assertion follows from the inequality

$$
\begin{aligned}
\left|\int_{\Omega}\left(\sqrt{1+\left|\nabla \eta_{n}\right|^{2}}-\sqrt{1+|\nabla \underline{u}|^{2}}\right)\right| & \leq \int_{\Omega}|| \nabla \eta_{n}|-| \nabla \underline{u}|| \\
& \leq \int_{\Omega}\left|\nabla \eta_{n}-\nabla \underline{u}\right| . .
\end{aligned}
$$

Using Lemma 3.7, Lemma 3.3, and Theorem 2.2 as the essential ingredients, we next prove that the minimizers of $\mathcal{J}_{\varepsilon}$ are convergent.

Theorem 3.8. Let $\phi^{\delta_{n}}$ satisfy

$$
\left\|\phi^{\delta_{n}}-\phi\right\|_{L^{1}(\partial \Omega)} \leq \delta_{n} \rightarrow 0 .
$$

Let $\eta_{n} \in W^{2, s}(\Omega)$ and $\rho_{n}$ be chosen as in Lemma 3.7, and let $\left\{\varepsilon_{n}\right\}=$ $\left\{\left(\varepsilon_{1, n}, \varepsilon_{2, n}\right)\right\}$ be a sequence with positive numbers $\varepsilon_{i, n}, i=1,2$, such that $\varepsilon_{2, n}\left\|\eta_{n}\right\|_{W^{2, s}(\Omega)}^{2} \rightarrow 0$. Then, as

$$
\frac{\rho_{n}}{\varepsilon_{1, n}} \rightarrow 0, \text { and } \frac{\delta_{n}}{\varepsilon_{1, n}} \rightarrow 0,
$$

we have

$$
\int_{\Omega} \sqrt{1+\left|\nabla u_{\varepsilon_{n}}^{\delta_{n}}\right|^{2}}+\int_{\partial \Omega}\left|T u_{\varepsilon_{n}}^{\delta_{n}}-\phi^{\delta_{n}}\right| \rightarrow \int_{\Omega} \sqrt{1+|\nabla \underline{u}|^{2}}+\int_{\partial \Omega}\left|T \underline{u}-\phi^{\delta_{n}}\right|,
$$

where $\underline{u}$ is a minimal solution of the minimal surface problem. Moreover, each subsequence of $\left\{u_{\varepsilon_{n}}^{\delta_{n}}\right\}$ has a (weakly) convergent subsequence and the limit of every (weakly) convergent subsequence is a minimal solution, i.e., if we denote by $\left\{u_{\varepsilon_{n}}^{\delta_{n}}\right\}$ a (weakly) convergent subsequence, then

$$
\left\{u_{\varepsilon_{n}}^{\delta_{n}}\right\} \rightarrow \underline{u} \text { in } L^{p_{0}}(\Omega), \quad\left\{u_{\varepsilon_{n}}^{\delta_{n}}\right\} \rightarrow \underline{u} \text { in } L^{p}(\Omega), \quad 1 \leq p<p_{0}=\frac{d}{d-1} .
$$


Proof. The proof consists of verification of the assumptions of Theorem 2.2. For this the notation and definitions introduced after the statement of Proposition 3.2 (see Notation 1) are used. In addition we let $a(u, v):=$ $(u, v)_{W^{2, s}(\Omega)}$, where $(., .)_{W^{2, s}(\Omega)}$ denotes the inner product on the Sobolev space $W^{2, s}(\Omega)$ and

$$
j_{\varepsilon}(u)=\frac{1}{2} \mathrm{e}(\varepsilon) a(u, u)
$$

It follows from Lemma 3.7 and the assumptions of the theorem that there exist sequences $\left\{\eta_{n}\right\},\left\{\varepsilon_{n}\right\}$ which satisfy

$$
j_{\varepsilon_{n}}\left(\eta_{n}\right) \rightarrow 0 \quad \text { and }
$$

$\limsup _{n \in \mathbb{N}}\left\{k_{\varepsilon_{n}}\left(\eta_{n}\right)+\phi_{\varepsilon_{n}}\left(\eta_{n}, \phi\right)\right\}=\limsup _{n \in \mathbb{N}}\left\{k\left(\eta_{n}\right)+\Phi\left(\eta_{n}, \phi\right)\right\} \leq k(\underline{u})+\Phi(\underline{u}, \phi)$.

Using the triangle inequality it follows that $\Phi$ satisfies (5).

To verify the coercivity condition of assumption 8 for the functional $\mathcal{J}_{\varepsilon}$, we first prove that for all $u \in W^{1,1}(\Omega)$

$$
\int_{\Omega}|u| \leq \hat{C}\left\{\int_{\Omega}|\nabla u|+\int_{\partial \Omega}|T u|\right\},
$$

where $\hat{C}_{1}$ is a constant independent of $u$. One can show that $T u \in L^{1}(\partial \Omega)$ for each $u \in W^{1,1}(\Omega)$ (see, for example, [6]). Moreover, for every $T u \in L^{1}(\partial \Omega)$ there exists (see e.g. [8]) a function $f \in W^{1,1}(\Omega)$, which satisfies $T f=T u$, and

$$
\|f\|_{W^{1,1}(\Omega)} \leq \hat{C}_{2}\|T u\|_{L^{1}(\partial \Omega)}
$$

where the constant $\hat{C}_{2}$ is independent of $T u$ and $f$. Since $T f=T u$ on $\partial \Omega$, it follows from Sobolev's inequality (see e.g. page 122 in [5]) that

$$
\int_{\Omega}|f-u| \leq \hat{C}_{1} \int_{\Omega}|\nabla f-\nabla u|,
$$

where $\hat{C}_{1}$ is a constant independent from $f$ and $u$. Consequently

$$
\begin{aligned}
\int_{\Omega}|u| & \leq \hat{C}_{1} \int_{\Omega}\{|\nabla u|+|\nabla f|+|f|\} \\
& \leq \hat{C}_{1} \int_{\Omega}|\nabla u|+\hat{C}_{1} \hat{C}_{2}\|T u\|_{L^{1}(\partial \Omega)} .
\end{aligned}
$$


With the notation $\hat{C}:=\max \left\{\hat{C}_{1}, \hat{C}_{1} \hat{C}_{2}\right\}$ it follows from above that

$$
\begin{aligned}
\|u\|_{W^{1,1}(\Omega)} & \leq(\hat{C}+1) \int_{\Omega}|\nabla u|+\hat{C}\|T u\|_{L^{1}(\partial \Omega)} \\
& \leq(\hat{C}+1) \int_{\Omega} \sqrt{1+|\nabla u|^{2}}+\hat{C}\left\|T u-\phi^{\delta}\right\|_{L^{1}(\partial \Omega)}+\hat{C}\left\|\phi^{\delta}\right\|_{L^{1}(\partial \Omega)} \\
& \leq(\hat{C}+1) \mathcal{J}_{\varepsilon}(u)+\hat{C}\left\|\phi^{\delta}\right\|_{L^{1}(\partial \Omega)},
\end{aligned}
$$

which shows that assumption 8 is satisfied.

In the case $1<p<\frac{d}{d-1}$, the embedding of $W^{1,1}(\Omega)$ into $L^{p}(\Omega)$ is compact and from Lemma 3.3 it follows that $\Phi(v, \phi)+k(v)$ (for fixed $\phi \in L^{1}(\partial \Omega)$ ) is lower semicontinuous on $\mathrm{BV}(\Omega)$ with respect to the norm on $L^{p}(\Omega)$, i.e., assumption 9 is satisfied. It then follows that there exists a minimizer of $\Phi(v, \phi)+k(v)$, since $\Phi$ and $k$ are positive, so assumption 6 is satisfied. Therefore, Theorem 2.2 is applicable, hence $\left\{u_{\varepsilon_{n}}^{\delta_{n}}\right\}$ has a subsequence which is convergent to a solution of the Dirichlet problem for the minimal surface problem with respect to the $L^{p}(\Omega)$-norm.

Now we show that the (weak) limit of each subsequence is a minimal solution of the minimal surface problem. Let $\left\{u_{\varepsilon_{n}}^{\delta_{n}}\right\}$ be a convergent subsequence in $L^{1}(\Omega)$ to $\hat{u}$ - note that $\left\{u_{\varepsilon_{n}}^{\delta_{n}}\right\}$ converges weakly to $\hat{u}$ in $L^{p}(\Omega), p=\frac{d}{d-1}$ and strongly in $L^{1}(\Omega)$. Let $\underline{u}$ be a minimal solution of the minimal surface problem. Then by the definition of $u_{\varepsilon_{n}}^{\delta_{n}}$, it follows that

$$
\begin{aligned}
& \varepsilon_{1, n} \varepsilon_{2, n} a\left(u_{\varepsilon_{n}}^{\delta_{n}}, u_{\varepsilon_{n}}^{\delta_{n}}\right)+\varepsilon_{1, n} \int_{\Omega}\left|u_{\varepsilon_{n}}^{\delta_{n}}\right|+\int_{\Omega} \sqrt{1+|\nabla \underline{u}|^{2}}+\int_{\partial \Omega}|T \underline{u}-\phi| \\
& \leq \quad \varepsilon_{1, n} \varepsilon_{2, n} a\left(u_{\varepsilon_{n}}^{\delta_{n}}, u_{\varepsilon_{n}}^{\delta_{n}}\right)+\varepsilon_{1, n} \int_{\Omega}\left|u_{\varepsilon_{n}}^{\delta_{n}}\right|+\int_{\Omega} \sqrt{1+\left|\nabla u_{\varepsilon_{n}}\right|^{2}}+\int_{\partial \Omega}\left|T u_{\varepsilon_{n}}^{\delta_{n}}-\phi\right| \\
& \leq \quad \varepsilon_{1, n} \varepsilon_{2, n} a\left(u_{\varepsilon_{n}}^{\delta_{n}}, u_{\varepsilon_{n}}^{\delta_{n}}\right)+\varepsilon_{1, n} \int_{\Omega}\left|u_{\varepsilon_{n}}^{\delta_{n}}\right|+\int_{\Omega} \sqrt{1+\left|\nabla u_{\varepsilon_{n}}\right|^{2}}+\int_{\partial \Omega}\left|T u_{\varepsilon_{n}}^{\delta_{n}}-\phi^{\delta_{n}}\right| \\
& \quad+\quad \delta_{n} \operatorname{meas}(\partial \Omega) \\
& \leq \quad \varepsilon_{1, n} \varepsilon_{2, n} a\left(\eta_{n}, \eta_{n}\right)+\varepsilon_{1, n} \int_{\Omega}\left|\eta_{n}\right|+\int_{\Omega} \sqrt{1+\left|\nabla \eta_{n}\right|^{2}}+\int_{\partial \Omega}\left|T \eta_{n}-\phi^{\delta_{n}}\right| \\
& \quad+\quad \delta_{n} \operatorname{meas}(\partial \Omega) \\
& \leq \quad \varepsilon_{1, n} \varepsilon_{2, n} a\left(\eta_{n}, \eta_{n}\right)+\varepsilon_{1, n} \int_{\Omega}\left|\eta_{n}\right|+\int_{\Omega} \sqrt{1+|\nabla \underline{u}|^{2}}+\int_{\partial \Omega}\left|T \underline{u}-\phi^{\delta_{n}}\right| \\
& \quad+\quad \rho_{n}+\delta_{n} \operatorname{meas}(\partial \Omega),
\end{aligned}
$$


where $\rho_{n}$ is as in Lemma 3.7. Since as $\delta_{n} \rightarrow 0$

$$
\varepsilon_{1, n} \varepsilon_{2, n} a\left(\eta_{n}, \eta_{n}\right)+\varepsilon_{1, n} \int_{\Omega}\left|\eta_{n}\right|+\rho_{n}+\delta_{n} \operatorname{meas}(\partial \Omega) \rightarrow 0,
$$

it follows using the third and fifth links of the above chain of inequalities that

$$
\begin{gathered}
\limsup _{n \in \mathbb{N}}\left\{\int_{\Omega} \sqrt{1+\left|\nabla u_{\varepsilon_{n}}^{\delta_{n}}\right|^{2}}+\int_{\partial \Omega}\left|T u_{\varepsilon_{n}}^{\delta_{n}}-\phi^{\delta_{n}}\right|\right\} \\
\leq \int_{\Omega} \sqrt{1+|\nabla \underline{u}|^{2}}+\int_{\partial \Omega}\left|T \underline{u}-\phi^{\delta_{n}}\right| .
\end{gathered}
$$

Using the first and third links of the above chain of inequalities it follows that

$$
\begin{aligned}
& \limsup _{n \in \mathbb{N}}\left\{\varepsilon_{2, n}\left\|u_{\varepsilon_{n}}^{\delta_{n}}\right\|_{W^{2, s}(\Omega)}^{2}+\int_{\Omega}\left|u_{\varepsilon_{n}}^{\delta_{n}}\right|\right\} \\
& \leq \lim _{n \rightarrow \infty}\left\{\varepsilon_{2, n}\left\|\eta_{n}\right\|_{W^{2, s}(\Omega)}^{2}+\int_{\Omega}\left|\eta_{n}\right|+\frac{\rho_{n}+2 \delta_{n} \operatorname{meas}(\partial \Omega)}{\varepsilon_{1, n}}\right\} \\
& \leq \int_{\Omega}|\underline{u}| .
\end{aligned}
$$

Finally, since $\left\{u_{\varepsilon_{n}}^{\delta_{n}}\right\} \rightarrow \hat{u}$ in $L^{1}(\Omega)$, we obtain

$$
\|\hat{u}\|_{L^{1}(\Omega)} \leq \liminf \left\|u_{\varepsilon_{n}}^{\delta_{n}}\right\|_{L^{1}(\Omega)} \leq \lim \sup \left\|\eta_{n}\right\|_{L^{1}(\Omega)} \leq\|\underline{u}\|_{L^{1}(\Omega)} .
$$

This shows that $\hat{u}$ is a minimal solution of the minimal surface problem.

\section{AN ITERATIVE IMPLEMENTATION FOR THE STABLE NUMERICAL SOLUTION OF A MINIMAL SURFACE PROBLEM}

Uzawa's algorithm and the augmented Lagrangian technique have been found to be efficient procedures for solving minimization problems for nondifferentiable functionals (see e.g. Glowinski [9]). The goal of this section is to characterize the minimizer of $\mathcal{J}_{\varepsilon}$ by Lagrange multipliers and use this characterization to compute the minimizer of $\mathcal{J}_{\varepsilon}$ via Uzawa's algorithm. Let $l, \Phi$ be functionals on $W^{2, s}(\Omega)$ defined by

$$
l(v)=\varepsilon_{1} \int_{\Omega}|v|, \quad \Phi(v)=\int_{\partial \Omega}\left|T v-\phi^{\delta}\right|
$$

where $\varepsilon_{1}$ is a fixed positive number, $T$ is the trace operator, and $\phi^{\delta} \in L^{2}(\partial \Omega)$. The functional $l, \Phi$, and $k$ (as introduced in Notation 1 following Proposition 
3.2) are such that the functional $\kappa:=k+l+\Phi$ is a lower semicontinuous, proper convex functional.

Let $a(.,)=.(., .)_{W^{2, s}(\Omega)}$. It is well known (see e.g. [9]) that if $\kappa$ satisfy the above property, then the minimizer of

$$
\mathcal{J}_{\varepsilon}(v):=\kappa(v)+\frac{1}{2} \varepsilon_{1} \varepsilon_{2} a(v, v)
$$

is unique and can be characterized as the a unique solution of the variational inequality

$$
\varepsilon_{1} \varepsilon_{2} a(u, v-u)+\kappa(v)-\kappa(u) \geq 0 .
$$

To characterize in a simpler way the solution of (9) and thus the minimizing element of $\mathcal{J}_{\varepsilon}$ we use a further assumption on $\Omega$. We tacitly assume that $\Omega$ is $C^{m}$-regular, where $m \geq s$ (and $s$ is the index of the Sobolev space $W^{2, s}(\Omega)$ ). Then each point $x$ in a $\rho$-neighborhood of $\partial \Omega, \mathcal{U}_{\rho}:=\{y \in \Omega: d(y, \partial \Omega)<\rho\}$ can be represented in a unique way by the following formula $x=x_{0}-\lambda n\left(x_{0}\right)$, where $x_{0}$ is the point of minimal distance on the boundary to $x, n\left(x_{0}\right)$ is the normal vector on $\partial \Omega(\|n\|=1)$, and $\lambda$ is a positive number.

If $\psi \in C^{m}(\partial \Omega)$, then the function

$$
f(x):=\left\{\begin{array}{rl}
\left(1-\frac{d(x, \partial \Omega)}{\rho}\right)^{4 m} \psi\left(x_{0}\right) & x \in \mathcal{U}_{\rho} \\
0 & x \in \Omega-\mathcal{U}_{\rho}
\end{array}\right.
$$

satisfies $f \in C^{m}(\bar{\Omega}), \int_{\Omega}|f|^{2} \leq \int_{1-\rho}^{1} C \int_{\partial \Omega}|\psi|^{2}$, where $C$ is a constant.

Since each boundary data $\phi \in L^{2}(\partial \Omega)$ can be approximated by a sequence $\left\{v_{n}\right\} \in C^{m}(\partial \Omega)$ with respect to the $L^{2}(\partial \Omega)$-norm, there exists a sequence $\left\{f_{n}\right\} \in C^{m}(\bar{\Omega})$ such that

$$
\int_{\Omega}\left|f_{n}\right|^{2} \rightarrow 0, \quad \int_{\partial \Omega}\left|T f_{n}-\phi\right| \rightarrow 0 .
$$

We summarize this result in the next lemma:

Lemma 4.1. Let $\Omega$ be a $C^{m}$-regular bounded domain and $\phi^{\delta} \in L^{2}(\partial \Omega)$. Then there exists a sequence of functions $g_{n} \in W^{2, s}(\Omega)$ such that

$$
\left\|T g_{n}-\phi^{\delta}\right\|_{L^{2}(\partial \Omega)} \rightarrow 0 \text { and }\left\|g_{n}\right\|_{L^{2}(\Omega)} \rightarrow 0 \text {. }
$$

Here $T g_{n}$ denotes the trace of $g_{n} \in W^{2, s}(\Omega)$. 
Let

$$
\Lambda:=\left\{\mu \in L^{2}(\partial \Omega):|\mu(x)| \leq 1 \text { a.e. on } \partial \Omega\right\}
$$

and

$$
\hat{\Lambda}:=\left\{\hat{\mu} \in L^{2}(\Omega):|\hat{\mu}(x)| \leq 1 \text { a.e. on } \Omega\right\} .
$$

Using this notation we state

Theorem 4.2. Let $\Omega$ be a $C^{m}$-regular bounded domain and $\phi^{\delta} \in L^{2}(\partial \Omega)$. Then the solution of (9) can be characterized by the existence of (Lagrange) multiplier functions $\lambda \in \Lambda, \hat{\lambda} \in \hat{\Lambda}$ such that

$$
\begin{gathered}
\varepsilon_{1} \varepsilon_{2} a(u, v)+\left(\frac{\nabla u}{\left.\sqrt{1+|\nabla u|^{2}}, \nabla v\right)_{L^{2}(\Omega)}+\varepsilon_{1} \int_{\Omega} \hat{\lambda} v+\int_{\partial \Omega} \lambda v=0 .}\right. \\
\lambda\left(u-\phi^{\delta}\right)=\left|u-\phi^{\delta}\right| \text { a.e. on } \partial \Omega, \\
\hat{\lambda} u=|u| \text { a.e. on } \Omega .
\end{gathered}
$$

Proof. For convenience in the proof we introduce the abbreviation

$$
b(u, v):=\varepsilon_{1} \varepsilon_{2} a(u, v)+\left(\frac{\nabla u}{\sqrt{1+|\nabla u|^{2}}}, \nabla v\right)_{L^{2}(\Omega)} .
$$

First we show that (9) implies (10) and (11): Let $\beta>0$, and define

$$
\begin{aligned}
\Phi_{\beta}(v) & :=\int_{\partial \Omega} \sqrt{\beta^{2}+\left|T v-\phi^{\delta}\right|^{2}}, \\
l_{\beta}(v) & :=\varepsilon_{1} \int_{\Omega} \sqrt{\beta^{2}+|v|^{2}}, \\
\kappa_{\beta}(v) & :=l_{\beta}(v)+\Phi_{\beta}(v)+k(v) .
\end{aligned}
$$

From the fact that the embedding of $W^{2, s}(\Omega)$ into $L^{p}(\Omega), 1 \leq p \leq \frac{d}{d-1}$, is compact, and the trace operator $T$ is compact from $W^{2, s}(\Omega)$ into $L^{1}(\partial \Omega)$, it follows using Lemma 3.3 that $\Phi_{\beta}, l_{\beta}$, and $k$ are lower semicontinuous on $W^{2, s}(\Omega)$. Since $\Phi_{\beta}, l_{\beta}$, and $k$ are proper convex functionals, the regularized variational inequality: For all $v \in W^{2, s}(\Omega)$

$$
\varepsilon_{1} \varepsilon_{2} a(w, v-w)+\kappa_{\beta}(v)-\kappa_{\beta}(w) \geq 0
$$

has a unique solution $u_{\beta} \in W^{2, s}(\Omega)$. In the following it is shown that $u_{\beta} \rightarrow u$, with respect to the $W^{2, s}(\Omega)$-norm.

From (9) and (12) we have

$$
\begin{array}{r}
\varepsilon_{1} \varepsilon_{2} a\left(u_{\beta}, u-u_{\beta}\right)+\kappa_{\beta}(u)-\kappa_{\beta}\left(u_{\beta}\right) \geq 0, \\
\varepsilon_{1} \varepsilon_{2} a\left(u, u_{\beta}-u\right)+\kappa\left(u_{\beta}\right)-\kappa(u) \geq 0 .
\end{array}
$$


Adding these two inequalities we obtain

$$
\varepsilon_{1} \varepsilon_{2} a\left(u-u_{\beta}, u-u_{\beta}\right)+\kappa_{\beta}\left(u_{\beta}\right)-\kappa\left(u_{\beta}\right) \leq \kappa_{\beta}(u)-\kappa(u) .
$$

From the inequality: For $t>0$

$$
0<\sqrt{t^{2}+\beta^{2}}-|t|=\frac{\beta^{2}}{\sqrt{t^{2}+\beta^{2}}+|t|} \leq \beta,
$$

and the fact that for any $v \in W^{2, s}(\Omega)$

$$
\kappa_{\beta}(v)-\kappa(v)=\left(\Phi_{\beta}(v)-\Phi(v)\right)+\left(l_{\beta}(v)-l(v)\right),
$$

it follows that

$$
0<\kappa_{\beta}(v)-\kappa(v) \leq \beta(\operatorname{meas}(\partial \Omega)+\operatorname{meas}(\Omega)) .
$$

The last inequality together with (13) implies

$$
\left\|u-u_{\beta}\right\|_{W^{2, s}(\Omega)} \leq\left\{(\operatorname{meas}(\partial \Omega)+\operatorname{meas}(\Omega)) \frac{\beta}{\varepsilon_{1} \varepsilon_{2}}\right\}^{(1 / 2)}
$$

which shows that $u_{\beta} \rightarrow u$ (strongly in $W^{2, s}(\Omega)$ ), as $\beta \rightarrow 0$.

Next we prove that $k$ has a one-sided Gateaux variation. Let $u, h \in W^{2, s}(\Omega)$.

Then the following holds:

$$
\begin{aligned}
& \lim _{t \rightarrow 0^{+}} \int_{\Omega}\left(\frac{\sqrt{1+|\nabla(u+t h)|^{2}}-\sqrt{1+|\nabla u|^{2}}}{t}\right) \\
& =\int_{\Omega} \frac{\nabla u \nabla h}{\sqrt{1+|\nabla u|^{2}}} \\
& -\lim _{t \rightarrow 0^{+}} \int_{\Omega} \nabla u \nabla h\left(\frac{\sqrt{1+|\nabla(u+t h)|^{2}}-\sqrt{1+|\nabla u|^{2}}}{\sqrt{1+|\nabla u|^{2}}\left(\sqrt{1+|\nabla(u+t h)|^{2}}+\sqrt{1+|\nabla u|^{2}}\right)}\right) \\
& =\int_{\Omega} \frac{\Omega}{\nabla u \nabla h} \frac{\sqrt{1+|\nabla u|^{2}}}{\sqrt{1+v^{2}}} \\
& -\lim _{t \rightarrow 0^{+}} \int_{\Omega} \nabla u \nabla h\left(\frac{|\nabla(u+t h)|^{2}-|\nabla u|^{2}}{\sqrt{1+|\nabla u|^{2}}\left(\sqrt{1+|\nabla(u+t h)|^{2}}+\sqrt{1+|\nabla u|^{2}}\right)^{2}}\right) .
\end{aligned}
$$


We now show that the second term in the preceding equality is zero. This follows from the following estimates:

$$
\begin{aligned}
& \left|\int_{\Omega} \nabla u \nabla h\left(\frac{|\nabla(u+t h)|^{2}-|\nabla u|^{2}}{\sqrt{1+|\nabla u|^{2}}\left(\sqrt{1+|\nabla(u+t h)|^{2}} \sqrt{1+|\nabla u|^{2}}\right)^{2}}\right)\right| \\
& \leq \int_{\Omega} \frac{|\nabla u||\nabla h||\nabla(u+t h)-\nabla u||\nabla(u+t h)+\nabla u|}{\sqrt{1+|\nabla u|^{2}}\left(\sqrt{1+|\nabla(u+t h)|^{2}}+\sqrt{1+|\nabla u|^{2}}\right)^{2}} \\
& \leq \int_{\Omega} t|\nabla h|^{2} .
\end{aligned}
$$

It then follows that $k$ is one-sided Gateaux differentiable on $W^{2, s}(\Omega)$, and the one-sided Gataux differential is given by

$$
k^{\prime}(u) h:=\int_{\Omega} \frac{\nabla u \nabla h}{\sqrt{1+|\nabla u|^{2}}} .
$$

Similarly it can be shown that $\phi_{\beta}$ and $l_{\beta}$ are one-sided Gateaux differentiable. Therefore $u_{\beta}$ satisfies

$$
b\left(u_{\beta}, v\right)+\left(i_{1, \beta}\left(u_{\beta}\right), T v\right)_{L^{2}(\partial \Omega)}+\left(i_{2, \beta}\left(u_{\beta}\right), v\right)_{L^{2}(\Omega)}=0,
$$

where

$$
i_{1, \beta}(u):=\frac{T u-\phi^{\delta}}{\sqrt{\beta^{2}+\left|T u-\phi^{\delta}\right|^{2}}}, \quad i_{2, \beta}(u):=\frac{u}{\sqrt{\beta^{2}+|u|^{2}}} .
$$

Since $\hat{p}_{\beta}:=i_{1, \beta}\left(u_{\beta}\right) \in \Lambda, \hat{p}_{\beta}:=i_{2, \beta}\left(u_{\beta}\right) \in \hat{\Lambda}$, the sets $\left\{p_{\beta}\right\}$ and $\left\{\hat{p}_{\beta}\right\}$ are weakly compact in $L^{2}(\partial \Omega)$ and $L^{2}(\Omega)$, respectively; i.e., there exists sequences, which will also be denoted by $p_{\beta}, \hat{p}_{\beta}$, such that

$$
p_{\beta} \rightarrow p \text { in } L^{2}(\partial \Omega), \quad \hat{p}_{\beta} \rightarrow \hat{p} \text { in } L^{2}(\Omega) \text { for } \beta \rightarrow 0 .
$$

The following estimate will be needed:

$$
\begin{aligned}
& \left|\int_{\Omega}\left(\frac{\nabla u_{\beta}}{\sqrt{1+\left|\nabla u_{\beta}\right|^{2}}}-\frac{\nabla u}{\sqrt{1+|\nabla u|^{2}}}\right) \nabla v\right| \\
& \leq\left|\int_{\Omega} \frac{\nabla u_{\beta}-\nabla u}{\sqrt{1+\left|\nabla u_{\beta}\right|^{2}}} \nabla v\right| \\
& +\int_{\Omega} \frac{|\nabla u \nabla v|\left|\nabla u_{\beta}-\nabla u\right|\left|\nabla u_{\beta}+\nabla u\right|}{\sqrt{1+\left|\nabla u_{\beta}\right|^{2}} \sqrt{1+|\nabla u|^{2}}\left(\sqrt{1+\left|\nabla u_{\beta}\right|^{2}}+\sqrt{1+|\nabla u|^{2}}\right)} \\
& \leq \quad C\|v\|_{W^{1,2}(\Omega)}\left\|u_{\beta}-u\right\|_{W^{1,2}(\Omega)} \text {. }
\end{aligned}
$$


It follows from (14), (15), (16) and the above estimate, letting $\beta \rightarrow 0$,

$$
b(u, v)+\int_{\partial \Omega} p T v+\varepsilon_{1} \int_{\Omega} \hat{p} v=0,
$$

which proves (10).

Let $g_{n}$ be as defined in Lemma 4.1. Then, since $p \in \Lambda, \hat{p} \in \hat{\Lambda}$, it follows from (17) that

$$
-b\left(u, g_{n}\right)=\int_{\partial \Omega} p T g_{n}+\varepsilon_{1} \int_{\Omega} \hat{p} g_{n} \rightarrow \int_{\partial \Omega} p \phi^{\delta} .
$$

Consequently from the definition of a minimizing element of the functional in (8) and the one-sided Gateaux differentiability of $k$ we obtain:

$$
\begin{aligned}
& 0 \leq \lim _{t \rightarrow 0^{+}}\left\{j_{\varepsilon}\left(u+t\left(g_{n}-u\right)\right)-j_{\varepsilon}(u)\right\} \\
& +\lim _{t \rightarrow 0^{+}}\left\{k\left(u+t\left(g_{n}-u\right)\right)-k(u)\right\} \\
& +\varepsilon_{1} \lim _{t \rightarrow 0^{+}} \int_{\Omega} \frac{\left|u+t\left(g_{n}-u\right)\right|-|u|}{t} \\
& +\lim _{t \rightarrow 0^{+}} \int_{\partial \Omega} \frac{\left|T\left(u+t\left(g_{n}-u\right)\right)-\phi^{\delta}\right|-\left|T u-\phi^{\delta}\right|}{t} \\
& \leq b\left(u, g_{n}-u\right) \\
& +\varepsilon_{1} \lim _{t \rightarrow 0^{+}} \int_{\Omega} \frac{\left|u+t\left(g_{n}-u\right)\right|-|u|}{t} \\
& +\lim _{t \rightarrow 0^{+}} \int_{\partial \Omega} \frac{\left|T\left(u+t\left(g_{n}-u\right)\right)-\phi^{\delta}\right|-\left|T u-\phi^{\delta}\right|}{t} \\
& \leq b\left(u, g_{n}-u\right) \\
& +\varepsilon_{1} \lim _{t \rightarrow 0^{+}} \int_{\Omega} \frac{|u-t u|-|u|+t\left|g_{n}\right|}{t} \\
& +\lim _{t \rightarrow 0^{+}} \int_{\partial \Omega} \frac{\left|T\left(u+t\left(\phi^{\delta}-u\right)\right)-\phi^{\delta}\right|+t\left|T g^{n}-\phi^{\delta}\right|-\left|T u-\phi^{\delta}\right|}{t} \\
& \leq b\left(u, g_{n}-u\right)-\varepsilon_{1}\|u\|_{L^{1}(\Omega)}+\varepsilon_{1}\left\|g_{n}\right\|_{L^{1}(\Omega)} \\
& -\left\|\phi^{\delta}-T u\right\|_{L^{1}(\partial \Omega)}+\left\|\phi^{\delta}-T g_{n}\right\|_{L^{1}(\partial \Omega)} .
\end{aligned}
$$

Taking the limit as $n \rightarrow \infty$ it follows from (17):

$$
\varepsilon_{1}\|u\|_{L^{1}(\Omega)}+\left\|\phi^{\delta}-T u\right\|_{L^{1}(\partial \Omega)} \leq b\left(u, g_{n}-u\right) \rightarrow \int_{\partial \Omega} p\left(T u-\phi^{\delta}\right)+\varepsilon_{1} \int_{\Omega} \hat{p} u,
$$


which is possible only if $p\left(T u-\phi^{\delta}\right)=\left|T u-\phi^{\delta}\right|$ almost everywhere in $\partial \Omega$ and $\hat{p} u=|u|$ almost everywhere in $\Omega$. To prove the necessity of (10) and (11), we first invoke the convexity of the function $\sqrt{1+|x|^{2}}$ to obtain

$$
\int_{\Omega} \sqrt{1+|\nabla v|^{2}}-\int_{\Omega} \sqrt{1+|\nabla u|^{2}} \geq\left(\frac{\nabla u}{\sqrt{1+|\nabla u|^{2}}}, \nabla(v-u)\right) .
$$

Then, it follows from (10) that for any $v \in W^{2, s}(\Omega)$

$$
\begin{aligned}
& \varepsilon_{1} \varepsilon_{2} a(u, v-u)+k(v)-k(u)+ \\
& \varepsilon_{1} \int_{\Omega} \hat{p}(v-u)+\int_{\partial \Omega} p(T v-T u) \\
& \quad \geq 0 .
\end{aligned}
$$

Since

$$
\begin{aligned}
\int_{\partial \Omega} p\left(T u-\phi^{\delta}\right) & =\int_{\partial \Omega}\left|T u-\phi^{\delta}\right|=\Phi(u), \\
\int_{\partial \Omega} p\left(T v-\phi^{\delta}\right) & \leq \int_{\partial \Omega}|p|\left|T v-\phi^{\delta}\right| \leq \int_{\partial \Omega}\left|T v-\phi^{\delta}\right|=\Phi(v),
\end{aligned}
$$

and

$$
\begin{aligned}
\varepsilon_{1} \int_{\Omega} \hat{p} u & =\varepsilon_{1}\|u\|_{L^{1}(\Omega)} \\
\varepsilon_{1} \int_{\Omega} \hat{p} v & \leq \varepsilon_{1} \int_{\Omega}\left|\hat{p}\left\|v \mid \leq \varepsilon_{1}\right\| v \|_{L^{1}(\Omega)}\right.
\end{aligned}
$$

we find

$$
\begin{aligned}
\int_{\partial \Omega} p(T v-T u) & =\int_{\partial \Omega} p\left(T v-\phi^{\delta}\right)-\int_{\partial \Omega} p\left(T u-\phi^{\delta}\right) \\
& \leq \Phi(v)-\Phi(u), \\
\varepsilon_{1} \int_{\Omega} \hat{p}(v-u) & \leq \varepsilon_{1}\left(\|v\|_{L^{1}(\Omega)}-\|u\|_{L^{1}(\Omega)}\right) .
\end{aligned}
$$

Moreover, from the definition of $a(.,$.$) it follows that$

$$
\begin{aligned}
& \frac{1}{2}\left(\varepsilon_{1} \varepsilon_{2} a(v, v)-\varepsilon_{1} \varepsilon_{2} a(u, u)\right) \\
= & \varepsilon_{1} \varepsilon_{2} a(u, v-u)+\frac{1}{2} \varepsilon_{1} \varepsilon_{2} a(v-u, v-u) \\
\geq & \varepsilon_{1} \varepsilon_{2} a(u, v-u) .
\end{aligned}
$$


Using the estimates (19) and (20) in (18), we obtain

$$
\begin{aligned}
& \frac{1}{2} \varepsilon_{1} \varepsilon_{2} a(v, v)+\Phi(v)+\varepsilon_{1}\|v\|_{L^{1}(\Omega)}+k(v) \\
& -\frac{1}{2} \varepsilon_{1} \varepsilon_{2} a(u, u)-\Phi(u)-\varepsilon_{1}\|u\|_{L^{1}(\Omega)}-k(u) \geq 0,
\end{aligned}
$$

which shows that $u$ minimizes $\mathcal{J}_{\varepsilon}$.

The technique used in the above proof requires the boundary data $\phi^{\delta}$ to be in $L^{2}(\partial \Omega)$, although this assumption was not necessary in the general results in Section 3. This requirement is used to deduce the convergence of the integrals $\int_{\partial \Omega} p_{\beta} T v, \int_{\partial \Omega} p_{\beta} T g_{n}$ from the weak convergence of $p_{\beta} \rightarrow p$ in $L^{2}(\partial \Omega)($ cf. (16)) .

In the following we show that Uzawa's algorithm can be used for the solution of the problem (10) - (11). Uzawa's algorithm is defined inductively as follows:

Definition 4.3. Let $p_{0} \in \Lambda, \hat{p}_{0} \in \hat{\Lambda}$, e.g., $p_{0}=0$ and $\hat{p}_{0}=0$. Moreover, let

$$
\begin{aligned}
p_{k+1}=P\left(p_{k}+\rho\left(T u_{k}-\phi^{\delta}\right)\right) & :=\frac{p_{k}+\rho\left(T u_{k}-\phi^{\delta}\right)}{\max \left\{1,\left|p_{k}+\rho\left(T u_{k}-\phi^{\delta}\right)\right|\right\}}, \\
\hat{p}_{k+1}=\hat{P}\left(\hat{p}_{k}+\varepsilon_{1} \rho u_{k}\right): & =\frac{\hat{p}_{k}+\varepsilon_{1} \rho u_{k}}{\max \left\{1,\left|\hat{p}_{k}+\varepsilon_{1} \rho u_{k}\right|\right\}},
\end{aligned}
$$

where $u_{k}$ denotes the solution of (10) for given parameters $p_{k} \in \Lambda, \hat{p}_{k} \in \hat{\Lambda}$.

We next show that Uzawa's algorithm is convergent, if the relaxation parameter is chosen appropriately:

\section{Theorem 4.4. If}

$$
\rho<\frac{\varepsilon_{1} \varepsilon_{2}}{\|T\|^{2}+\varepsilon_{1}^{2}}
$$

where $\|T\|$ is the norm of the trace operator from $W^{2, s}(\Omega)$ into $L^{2}(\partial \Omega)$, then

$$
\lim _{k \rightarrow \infty}\left\|u_{k}-u\right\|_{W^{2, s}(\Omega)}=0
$$

where $u$ minimizes $\mathcal{J}_{\varepsilon}$.

Proof. Let $\{\underline{u}, p, \hat{p}\}$ satisfy (10) and (11). We claim that

$$
p=P\left(p+\rho\left(T \underline{u}-\phi^{\delta}\right)\right), \quad \hat{p}=\hat{P}\left(\hat{p}+\varepsilon_{1} \rho \underline{u}\right) .
$$

We prove the first equality; the proof of the second equality is similar. If $\left|T \underline{u}-\phi^{\delta}\right|=0$ then $\left|p+\rho\left(T \underline{u}-\phi^{\delta}\right)\right| \leq 1$ and consequently $P\left(p+\rho\left(T \underline{u}-\phi^{\delta}\right)\right)=p$. We now assume that $\left|T \underline{u}-\phi^{\delta}\right|>0$. Then from the 
facts that $p \in \Lambda$ and $p\left(T \underline{u}-\phi^{\delta}\right)=\left|T \underline{u}-\phi^{\delta}\right|$, it follows that

$$
p=\frac{T \underline{u}-\phi^{\delta}}{\left|T \underline{u}-\phi^{\delta}\right|}
$$

Using this it follows from simple manipulations that $\left|p+\rho\left(T \underline{u}-\phi^{\delta}\right)\right|>1$, and consequently the assertion holds. Let

$$
\bar{u}_{k}:=T u_{k}-T \underline{u}, \quad \bar{p}_{k}:=p_{k}-p, \quad \overline{\hat{p}}_{k}:=\hat{p}_{k}-\hat{p} .
$$

Since the mappings $P, \hat{P}$ are contractions, we obtain

$$
\begin{aligned}
&\left\|\bar{p}_{k+1}\right\|_{L^{2}(\partial \Omega)}^{2} \leq\left\|P\left(p_{k}+\rho\left(T u_{k}-\phi^{\delta}\right)\right)-P\left(p+\rho\left(T \underline{u}-\phi^{\delta}\right)\right)\right\|_{L^{2}(\partial \Omega)}^{2} \\
& \leq\left\|\bar{p}_{k}+\rho \bar{u}_{k}\right\|_{L^{2}(\partial \Omega)}^{2} \\
& \leq\left\|\bar{p}_{k}\right\|_{L^{2}(\partial \Omega)}^{2}+2 \rho \int_{\partial \Omega} \bar{p}_{k} \bar{u}_{k}+\rho^{2} \int_{\partial \Omega}\left|\bar{u}_{k}\right|^{2} \\
&\left\|\overline{\hat{p}}_{k+1}\right\|_{L^{2}(\Omega)}^{2} \leq\left\|\overline{\hat{p}}_{k}\right\|_{L^{2}(\Omega)}^{2}+2 \varepsilon_{1} \rho \int_{\Omega} \overline{\hat{p}}_{k} \bar{u}_{k}+\varepsilon_{1}^{2} \rho^{2} \int_{\Omega}\left|\bar{u}_{k}\right|^{2} .
\end{aligned}
$$

From (10) it follows that

$$
\varepsilon_{1} \varepsilon_{2} a\left(\bar{u}_{k}, v\right)+\left(\frac{\nabla u_{k}}{\sqrt{1+\left|\nabla u_{k}\right|^{2}}}-\frac{\nabla \underline{u}}{\sqrt{1+|\nabla \underline{u}|^{2}}}, \nabla v\right)_{L^{2}(\Omega)}+\varepsilon_{1} \int_{\Omega} \overline{\hat{p}}_{k} v+\int_{\partial \Omega} \bar{p}_{k} T v=0
$$

from which we derive letting $v=\bar{u}_{k}$ :

$$
\begin{aligned}
& \varepsilon_{1} \varepsilon_{2} a\left(\bar{u}_{k}, \bar{u}_{k}\right)+\left(\frac{\nabla u_{k}}{\sqrt{1+\left|\nabla u_{k}\right|^{2}}}-\frac{\nabla \underline{u}}{\sqrt{1+|\nabla \underline{u}|^{2}}}, \nabla \bar{u}_{k}\right)_{L^{2}(\Omega)} \\
& \quad+\varepsilon_{1} \int_{\Omega} \overline{\hat{p}}_{k} \bar{u}_{k}+\int_{\partial \Omega} \bar{p}_{k} \bar{u}_{k} \\
& =0 .
\end{aligned}
$$


From (22), (23), and (24) we obtain

$$
\begin{aligned}
& \left(\left\|\bar{p}_{k}\right\|^{2}+\left\|\overline{\hat{p}}_{k}\right\|^{2}\right)-\left(\left\|\bar{p}_{k+1}\right\|^{2}+\left\|\overline{\hat{p}}_{k+1}\right\|^{2}\right) \\
& \geq-2 \rho \int_{\partial \Omega} \bar{p}_{k} \bar{u}_{k}-\rho^{2} \int_{\partial \Omega}\left|\bar{u}_{k}\right|^{2} \\
& \quad-2 \rho \varepsilon_{1} \int_{\Omega} \overline{\hat{p}}_{k} \bar{u}_{k}-\varepsilon_{1}^{2} \rho^{2} \int_{\Omega}\left|\bar{u}_{k}\right|^{2} \\
& =2 \rho \varepsilon_{1} \varepsilon_{2} a\left(\bar{u}_{k}, \bar{u}_{k}\right) \\
& \quad+2 \rho\left(\frac{\nabla u_{k}}{\left.\sqrt{1+\left|\nabla u_{k}\right|^{2}}-\frac{\nabla \underline{u}}{\sqrt{1+|\nabla \underline{u}|^{2}}}, \nabla \bar{u}_{k}\right)_{L^{2}(\Omega)}}\right. \\
& \quad-\rho^{2} \int_{\partial \Omega}\left|\bar{u}_{k}\right|^{2}-\varepsilon_{1}^{2} \rho^{2} \int_{\Omega}\left|\bar{u}_{k}\right|^{2} .
\end{aligned}
$$

Using the inequalities

$$
\left(\frac{\nabla u_{k}}{\sqrt{1+\left|\nabla u_{k}\right|^{2}}}-\frac{\nabla \underline{u}}{\sqrt{1+|\nabla \underline{u}|^{2}}}, \nabla \bar{u}_{k}\right)_{L^{2}(\Omega)} \geq 0
$$

(note that $\sqrt{1+|\nabla \cdot|^{2}}$ is convex), and

$$
\begin{aligned}
\rho^{2} \int_{\partial \Omega}\left|T \bar{u}_{k}\right|^{2}+\varepsilon_{1}^{2} \rho^{2} \int_{\Omega}\left|\bar{u}_{k}\right|^{2} & \leq \rho^{2}\left(\|T\|^{2}+\varepsilon_{1}^{2}\right)\left\|\bar{u}_{k}\right\|_{W^{2, s}(\Omega)}^{2} \\
& \leq \frac{\rho^{2}\left(\|T\|^{2}+\varepsilon_{1}^{2}\right)}{\varepsilon_{1} \varepsilon_{2}} \varepsilon_{1} \varepsilon_{2} a\left(\bar{u}_{k}, \bar{u}_{k}\right),
\end{aligned}
$$

it follows from $(21)$ and $(25)$ that the sequence $\left\{\left\|\bar{p}_{k}\right\|^{2}+\left\|\overline{\hat{p}}_{k}\right\|^{2}\right\}$ is strictly monotonically decreasing and bounded from below. Consequently it is convergent, and therefore it follows from $(25)$ that $\left\|\bar{u}_{k}\right\|_{W^{2, s}(\Omega)} \rightarrow 0$.

\section{REFERENCES}

[1] J. M. Ball, Convexity conditions and existence theorems in nonlinear elasticity, Arch. Rat. Mech. Anal. 63 (1977), 337-403.

[2] J. M. Ball and F. Murat, $W^{1, p}$ quasiconvexity and variational problems for multiple integrals, J. Funct. Anal. 58 (1984), 225 - 253.

[3] P. Concus, Numerical solution of Plateau's problem, Math. Comp. 17 (1967), 340 350.

[4] B. Dacorogna, Direct Methods in the Calculus of Variations, Springer, Berlin, 1989.

[5] I. Ekeland and R. Temam, Convex Analysis and Variational Problems, North Holland, Amsterdam, 1976.

[6] L. C. Evans and R. F. Gariepy, Measure Theory and Fine Properties of Functions, CRC-Press, Boca Raton, 1992.

[7] A. T. Fomenko, The Plateau Problem: Part I, Historical Survey; Part II, The Present State of the Theory, Gordon and Breach, New York, 1989. 
[8] E. Giusti, Minimal Surfaces and Functions of Bounded Variation, Birkhäuser, Boston, 1984.

[9] R. Glowinski, Numerical Methods for Nonlinear Variational Problems, Springer, New York, 1984.

[10] S. Hildebrandt and A. Tromba, Mathematics and Optimal Form, Sci. Amer. Library, W. H Freemann, New York, 1985.

[11] D. Hoffman, The computer-aided discovery of new embedded minimal surfaces, Math. Intelligencer, 7 (1987), No.3, 8-21.

[12] H. Jenkins and J. Serrin, The Dirichlet problem for the minimal surface problem in higher dimensions, J. Reine Angew. Math. 229 (1968), 170-187.

[13] C. Jouron, Resolution numérique du probléme de surfaces minima, Arch. Rat. Mech. Anal. 59, 311-348 (1975).

[14] J. L.Lions and E. Magenes, Non-Homogeneous Boundary Value Problems and Applications, Springer, New York, 1972.

[15] M. Z. Nashed and O. Scherzer, Stable approximation of nondifferentiable optimization problems with variational inequalities, in "Recent Developments in Optimization Theory and Nonlinear Analysis", (eds. Y. Censor and S. Reich), Contemp. Math., \#204, Amer. Math. Soc., Providence, 1997, 155-170.

[16] J. Nitsche, On new results in the theory of minimal surfaces, Bull. Amer. Math. Soc. 71 (1965), $195-270$.

[17] J. Nitsche, Introduction to Minimal Surfaces, Cambridge Univ. Press, Cambridge, 1989.

[18] R. Osserman, Global Properties of Minimal Surfaces, 2nd ed., Dover, New York, 1986.

[19] J. L. Troutman, Variational Calculus with Elementary Convexity, 2nd ed., Springer, New York, 1995.

\section{Department of Mathematical Sciences}

UNIVERSity of Delaware

Newark, DE 19716, U. S. A.

E-mail Address: nashed@math.udel. edu

INSTITUT FÜR INDUSTRIEMATHEMATIK

JOHANNES-KEPLER-UNIVERSITÄT

A-4040 LINZ

ÖSTERREICH / AUSTRIA

E-mail Address: scherzer@indmath.uni-linz.ac.at 


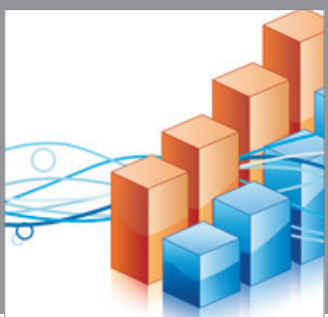

Advances in

Operations Research

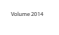

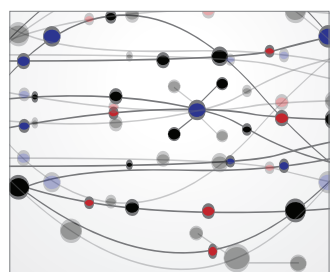

\section{The Scientific} World Journal
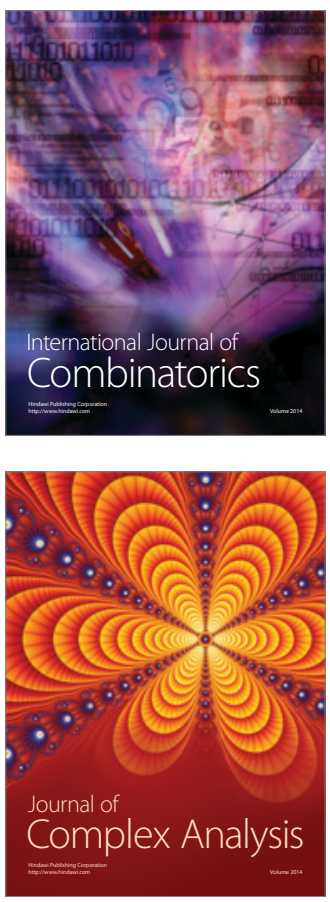

International Journal of

Mathematics and

Mathematical

Sciences
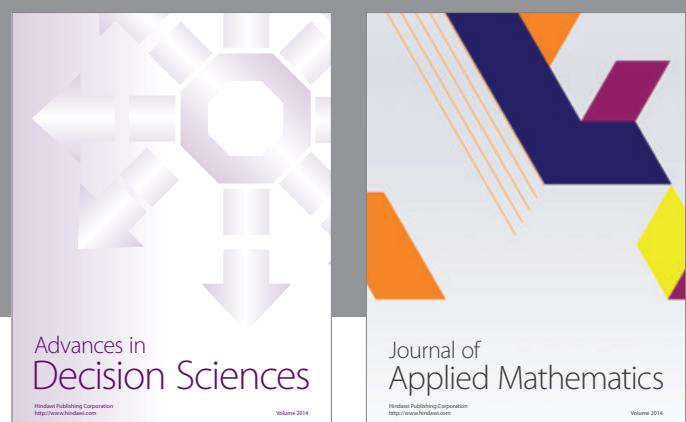

Journal of

Applied Mathematics
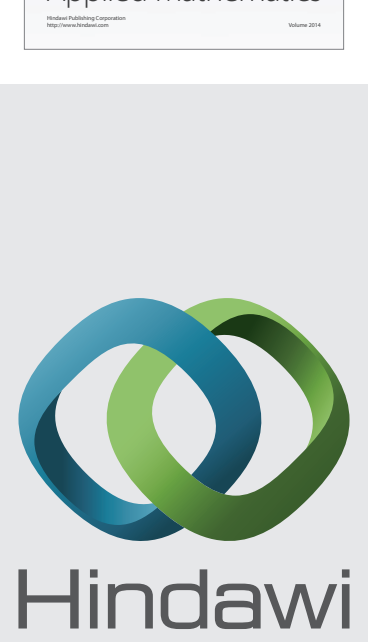

Submit your manuscripts at http://www.hindawi.com
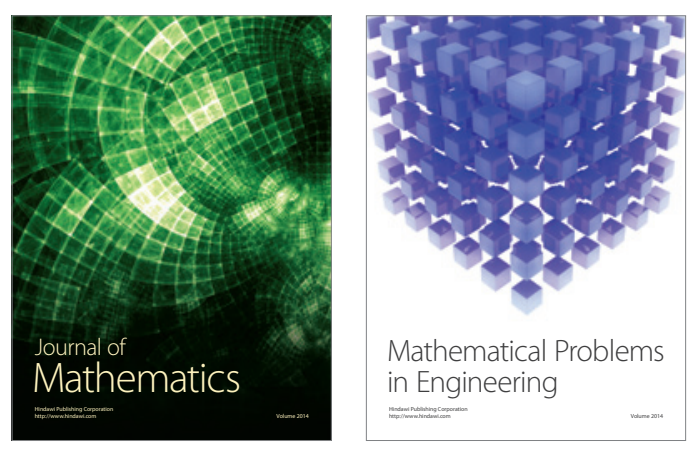

Mathematical Problems in Engineering
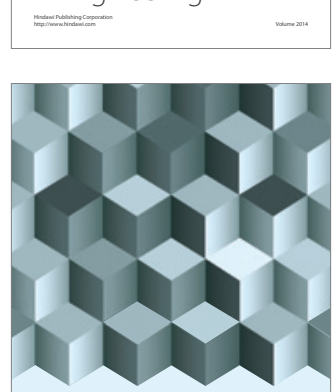

Journal of

Function Spaces
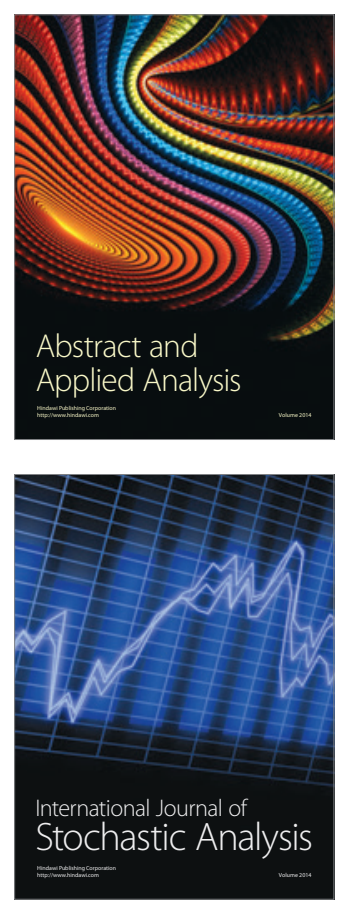

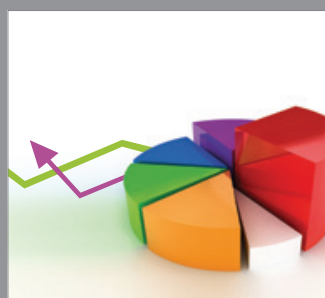

ournal of

Probability and Statistics

Promensencen
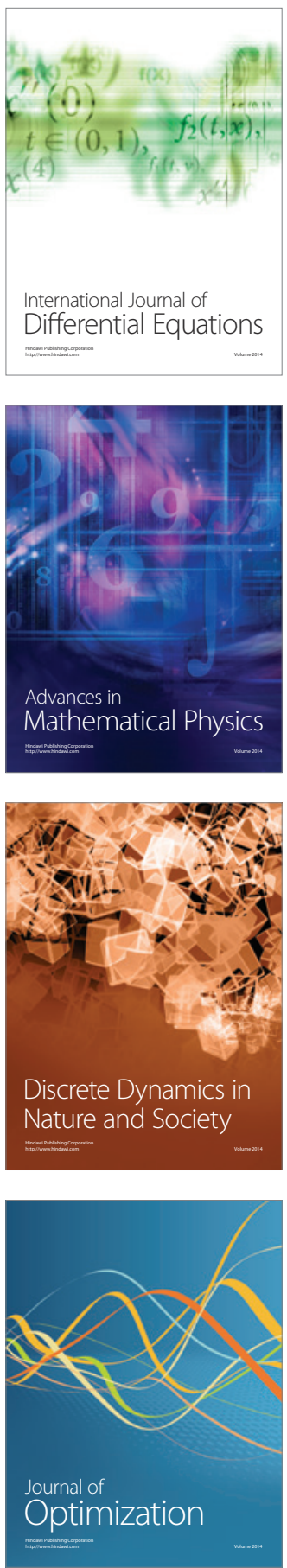